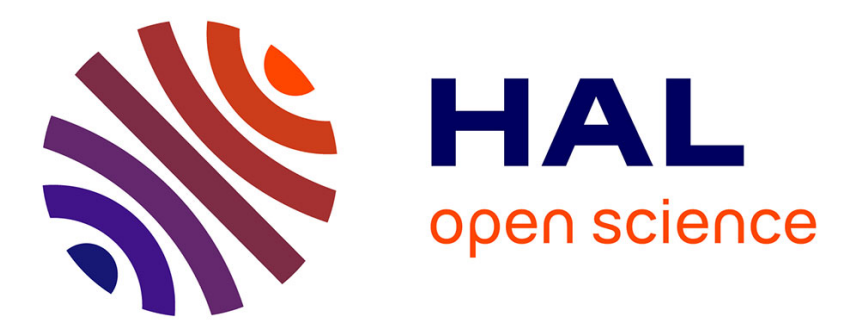

\title{
Three-dimensional Maxwell-Bloch calculation of the temporal profile of a seeded soft x-ray laser pulse
}

F. Tissandier, S Sebban, J Gautier, Ph Zeitoun, E Oliva, Antoine Rousse, G Maynard

\section{- To cite this version:}

F. Tissandier, S Sebban, J Gautier, Ph Zeitoun, E Oliva, et al.. Three-dimensional Maxwell-Bloch calculation of the temporal profile of a seeded soft x-ray laser pulse. Applied Physics Letters, 2012, 101, pp.251112. 10.1063/1.4773208 . hal-01165577

\section{HAL Id: hal-01165577 \\ https://hal-ensta-paris.archives-ouvertes.fr/hal-01165577}

Submitted on 19 Jun 2015

HAL is a multi-disciplinary open access archive for the deposit and dissemination of scientific research documents, whether they are published or not. The documents may come from teaching and research institutions in France or abroad, or from public or private research centers.
L'archive ouverte pluridisciplinaire HAL, est destinée au dépôt et à la diffusion de documents scientifiques de niveau recherche, publiés ou non, émanant des établissements d'enseignement et de recherche français ou étrangers, des laboratoires publics ou privés. 
Three-dimensional Maxwell-Bloch calculation of the temporal profile of a seeded soft x-ray laser pulse

F. Tissandier, ${ }^{1, \text { a) }}$ S. Sebban, ${ }^{1}$ J. Gautier, ${ }^{1}$ Ph. Zeitoun, ${ }^{1}$ E. Oliva, ${ }^{1}$ A. Rousse, ${ }^{1}$ and G. Maynard ${ }^{2}$

1) Laboratoire d'Optique Appliquée, ENSTA Paristech/Ecole

Polytechnique/CNRS UMR 7639, F-91761 Palaiseau Cedex,

France

${ }^{2)}$ Laboratoire de Physique des Gaz et des Plasmas, Université

Paris Sud / CNRS UMR 8578, F-91405 Orsay Cedex,

France

(Dated: 27 January 2014)

We present three-dimensional modeling of amplification of a high-order harmonic seed by a soft x-ray laser plasma. The time-dependent evolution of the x-ray signal is determined from a fully dynamic Maxwell-Bloch calculation. At high seed intensities, a simplified one-dimensional calculation leads to strong Rabi-like temporal oscillations of the output signal. However, such oscillations have not been observed experimentally. Our three-dimensional calculations demonstrate that this is due to spatial non-uniformities in the plasma gain that cause the Rabi oscillations to dampen dramatically. Large amplitude Rabi-like oscillations are expected to appear only in long and uniform plasma. Such targets require optimized guiding techniques.

a)Electronic mail: fabien.tissandier@ensta-paristech.fr 
There is a considerable interest in the development of high-brightness coherent radiation sources in the soft $\mathrm{x}$-ray region. While free-electron lasers offer unrivaled performances in terms of peak power and temporal resolution, there is a need for more compact and affordable alternative sources. High-order harmonic $(\mathrm{HOH})$ seeded plasma-based soft x-ray lasers are a promising complementary option.

The first experimental realization of such a source was performed in 1995 using a gallium plasma amplifier ${ }^{1}$, but the seed amplification was very low. Saturated amplification of a high-order harmonic in the soft x-ray region was not reached until 2004 in a nickel-like krypton plasma amplifier created by optical field ionization (OFI) ${ }^{2}$, and in 2006 in a neon-like titanium amplifier from a solid target ${ }^{3}$. Intense $(\mathrm{mJ})$, coherent and ultrashort (fs) soft x-ray pulses were expected but never actually generated. Various experimental studies reported the production of picosecond-duration soft x-ray pulses ${ }^{45}$ with up to $1 \mu \mathrm{J}$ energy $^{267}$. Such a long pulse duration is due to the very narrow bandwidth $(\mathrm{m} \AA)$ of the amplifier ${ }^{85}$, which does not allow the amplification of the whole harmonic seed bandwidth. In the temporal domain, it has also been shown ${ }^{9}$ that the amplified pulse exhibits a rather unusual profile due to the strong mismatch of the seed (femtosecond) and amplifier (picosecond) time scales.

In this paper, we provide a numerical study of the $\mathrm{HOH}$ amplification process by an OFI krypton plasma at a wavelength of $32.8 \mathrm{~nm}$ corresponding to the $4 \mathrm{~d}\left({ }^{1} \mathrm{~S}_{0}\right) \rightarrow 4 \mathrm{p}\left({ }^{1} \mathrm{P}_{1}\right)$ transition in $\mathrm{Kr}^{8+}$. In the amplification of spontaneous emission (ASE) regime, both the electric dipole and the population inversion are in a quasi-equilibrium with the emitted electric field variations. On the contrary, when the amplifier is seeded with a fs-duration external field, we must consider a full dynamic regime without any adiabatic approximation. A convenient formalism to study this regime is the use of two-level Maxwell-Bloch (MB) equations $^{91011}$.

In a first step, we consider a one-dimensional (1D) geometry, neglecting any transverse gradient. When considering $\mathrm{HOH}$ amplification in an OFI plasma, both spontaneous emission and absorption by the plasma can be safely neglected. Therefore, within the slowly varying envelope approximation, the Maxwell-Bloch equations for a 1D two-level amplifier at the resonant frequency $\omega$ reduce to ${ }^{12}$ :

$$
\left(\frac{\partial}{\partial z}+\frac{1}{c} \frac{\partial}{\partial t}\right) A=D
$$




$$
\begin{aligned}
& \frac{\partial D}{d t}=-\frac{D}{\tau_{d}}+\frac{Z_{0} \omega}{2 \hbar}|M|^{2} A W \\
& \frac{\partial W}{d t}=-\frac{W-W_{0}}{\tau_{g}}-\frac{3}{4 \hbar \omega}\left(A D^{*}+A^{*} D\right)
\end{aligned}
$$

$A(z, t)$ is the amplitude of the resonant soft x-ray field and $D(z, t)$ is the dipole moment of the medium. $W(z, t)$ represents the population inversion between the two lasing levels and $W_{0}(z, t)$ its initial ( $i$. e. just before the $\mathrm{HOH}$ injection) value. $Z_{0}$ is the wave impedance of free space and $|M|$ the dipole interaction matrix diagonal element modulus. $\tau_{g}$ and $\tau_{d}$ are respectively the gain recovery time (energy relaxation) and the dipole relaxation time (phase relaxation, mostly due to electron-ion collisions). Radiative losses occur on a nanosecond time scale and can be neglected. The ASE has an estimated intensity of $10^{3}-10^{4} \mathrm{~W} / \mathrm{cm}^{2}$, well below the external $\mathrm{HOH}$ field intensity $\left(10^{8}-10^{9} \mathrm{~W} / \mathrm{cm}^{2}\right)$; its contribution can be safely neglected.

The gain recovery time, dipole relaxation time, and initial population inversion have been calculated using the collisional-radiative time-dependent code OFI-0D ${ }^{13}$ aiming at determining the evolution of a krypton plasma created via OFI by a strong infrared field. Since the OFI process can be considered instantaneous compared to any other timescale involved here and in particular to the pulse duration, the state of the plasma after interaction with the pump pulse only depends on the maximum intensity of this pulse. The evolution of the plasma parameters, occurring on the ps time scale can then be written in OFI-0D as a function of the maximum intensity of the pump pulse. These functions are then introduced in a parametrized form in the MB equations, which are solved using the COFIXE-MB code ${ }^{14}$ following Kärtner MIT lecture course ${ }^{15}$. We typically use 10,000 calculations points per cm and the calculation time ranges from negligible to a few seconds. The calculations have been performed with a circular polarization for the pump field, with an intensity of $10^{17} \mathrm{~W} / \mathrm{cm}^{2}$, while the neutral krypton pressure is set to 30 mbar and the target length is limited to $5 \mathrm{~mm}$. The $\mathrm{HOH}$ pulse has a duration of $35 \mathrm{fs}$ and is focused at an intensity of $5 \times 10^{8} \mathrm{~W} / \mathrm{cm}^{2}$. It is injected at $t=0$, with a delay of $3 \mathrm{ps}$ behind the infrared pulse. This configuration corresponds to the maximum of soft x-ray output signal in the experimental data reported in $^{51617}$. From our calculations, we get $\tau_{g} \simeq \tau_{d}=2$ ps, whereas $W_{0}(t)$, plotted on Fig. 1-a (dashed line), has a peak value leading to a maximum gain of $170 \mathrm{~cm}^{-1}$.

The profile of the output pulse is given in Fig. 1-(b) for different amplification lengths. Since the duration of the $\mathrm{HOH}$ pulse is much shorter than the dipole relaxation time $\tau_{d}$, the 
$\mathrm{HOH}$ peak itself is barely amplified ${ }^{9}$. In this strongly non-adiabatic regime, the response of the amplifying medium to the $\mathrm{HOH}$ excitation takes place within a characteristic time $\tau_{d}$, much longer than the $\mathrm{HOH}$ peak duration. Stimulated emission then occurs behind the $\mathrm{HOH}$ peak ${ }^{9}$, giving rise to a longer, second pulse with a duration close to $\tau_{d}$ as can be seen for an amplification length of $2 \mathrm{~mm}$. Then at $3 \mathrm{~mm}$, this second pulse is further amplified and its duration is increased according to standard spectral gain narrowing. At a critical length close to $4 \mathrm{~mm}$, the temporal profile exhibits oscillating features. The evolution between 4 and $5 \mathrm{~mm}$ shows that both the amplitude and the frequency of these oscillations are increasing. This phenomenon is related to the well-known Rabi oscillations of the populations for two atomic states interacting with a resonant electromagnetic field ${ }^{18}$. The frequency of Rabi oscillations is given by $\Omega_{R}=\sqrt{I / I_{s} \tau_{g} \tau_{d}}$, where $I$ is the resonant field intensity and $I_{s}$ the saturation intensity of the amplifier. In our situation, the Rabi oscillations can appear only if $\Omega_{R} \tau_{d}>1$. Using $\tau_{g} \simeq \tau_{d}$ the intensity threshold for Rabi oscillations is therefore higher than $I_{s}$. In fact, the very fast evolution of the population inversion observed at $5 \mathrm{~mm}$ (Fig.1-(a)) indicates that, at this distance, the maximum intensity is indeed well above $I_{s}$.

Stimulation and amplification of Rabi oscillations offer strong potentialities for applications. In particular, during the amplification process, the duration of the first peak decreases while its intensity increases. As a consequence, a strong soft x-ray pulse with sub-ps duration can be generated by a cm-long plasma amplifier.

The temporal profile of a harmonic-seeded soft x-ray laser pulse has never been experimentally measured with a resolution high enough to allow direct observation of such an oscillatory structure. However, the temporal coherence measurement data presented in Ref. ${ }^{16}$ or in Ref. ${ }^{19}$ showed no evidence of large oscillating features. In order to clarify this issue, we performed more realistic calculations using experimental results as input data. To do so, we have solved the MB equations in a 3D $(\rho, \theta, z)$ geometry. Maxwell equation (Eq. 1) now takes into account the radial diffraction term and reads:

$$
\left(\frac{\partial}{\partial z}+\frac{1}{c} \frac{\partial}{\partial t}\right) A=\frac{i}{2 k} \nabla_{\rho}^{2} A+D
$$

where $k$ is the wave vector of the resonant field and $\nabla_{\rho}^{2}=\frac{\partial^{2}}{\partial \rho^{2}}+\frac{1}{\rho} \frac{\partial}{\partial \rho}+\frac{1}{\rho^{2}} \frac{\partial^{2}}{\partial \theta^{2}}$ is the transverse Laplacian. 3D effects have been included by projecting the physical quantities on a radial grid and on azimuthal modes using the general formula $A(\rho, \theta, z, t)=\sum_{\ell=-N}^{\ell=+N} A_{\ell}(\rho, z, t) e^{i \ell \theta}$. Our calculations show that accurate results can be obtained with no more than 11 modes, 
given by $2 N+1$. We typically use 100 grid points for radial values and 10,000 points per $\mathrm{cm}$ for longitudinal values. The code is not parallelized and a few hours of computer time are needed to perform a 3D calculation.

The injected $\mathrm{HOH}$ amplitude was determined from experimental data following the procedure detailed in Ref. ${ }^{17}$ and is then described in 3D. The amplifier geometry is calculated in a two-dimensional $(2 \mathrm{D})(\rho, z)$ geometry by the infrared propagation code OFI-PROP ${ }^{13}$, assuming a cylindrical symmetry of the pump beam. The amplification is calculated -as suggested by Eq. 4- in 3D. The plasma gain map, calculated for a focused pump intensity in vacuum of $10^{18} \mathrm{~W} / \mathrm{cm}^{2}$, is given in Fig. 2-(a). It can be seen that the amplifier is far from being homogeneous, a little shorter than $5 \mathrm{~mm}$, and exhibits a hollow near the axis due to over-ionization close to the maximum of the infrared intensity. The amplifier is thus radially strongly inhomogeneous. It is worth specifying that the $\mathrm{HOH}$ seed beam is assumed to be collinear to the infrared pump beam (and thus to the amplifier).

The temporal profile of the power of the output pulse at different amplification lengths up to $5 \mathrm{~mm}$ (longer than the amplifier length) is represented on Fig. 2-(b). That power is obtained by summing the contributions $P_{\ell}(z, t)$ of every mode $\ell$, which are obtained by radial integration over the whole beam cross section $P_{\ell}(z, t)=\int_{\rho} I_{\ell}(\rho, z, t) 2 \pi \rho d \rho=$ $\int_{\rho}\left|A_{\ell}(\rho, z, t)\right|^{2} 2 \pi \rho d \rho$. Due to the cylindrical symmetry of the amplifier and the good quality of the injected $\mathrm{HOH}$ beam, the output amplified soft x-ray beam exhibits a strong radial symmetry. In fact, only the $\ell=0, \pm 1$ modes yield a significant contribution to the output signal, and the $\ell=0$ mode accounts for more than $90 \%$ of the output intensity. Therefore we can conclude that 2D non-uniformities in the plasma, rather than the seed beam quality, play the dominant role to suppress Rabi oscillations.

The duration of the calculated amplified pulse (a few ps) is consistent with the $4.7 \mathrm{ps}$ value given in Ref. ${ }^{5}$, which determination was based on experimental gain duration and temporal coherence measurements. From Fig. 2-(b), we can observe that, using a more realistic 3D simulation, the strong oscillations predicted by the 1D calculations no longer exist. The main reason for the large difference between the 1D and the 3D results is that the average gain, over the $5 \mathrm{~mm}$-long target, is in fact smaller in the realistic calculations than in the ideal 1D case. The pump laser is indeed strongly refracted by the plasma it creates. The electronic density is higher near the propagation axis, causing defocusing of the pump beam. This defocusing adds a contribution to radial diffraction, leading to a 
significant reduction of the amplifying volume. The $1 \mathrm{D}$ calculation is relevant only at low pressures, below 10 mbar, at which defocusing by the plasma is very weak. However, low pressure targets leading to small amplification factor are not suitable for applications. At higher pressures, an optimized amplification factor is obtained by increasing the pump laser intensity. As shown in Fig. 2.(a), at such intensities, over-ionized domains appear close to the axis, leading to a strong reduction of the gain. Therefore, at pressures corresponding to a maximum of the amplification factor, the average gain cannot reach the $1 \mathrm{D}$ value over the whole target length.

Looking more closely at Fig. 2-(a), we can select some linear trajectories having a maximum value of the gain over more than $4 \mathrm{~mm}$. From Fig. 1-(b) we can deduce that the soft x-ray radiation following these trajectories will exhibit temporal oscillation. This can indeed be seen on Fig. 3 showing some intensity profiles $I(\rho, z, t)$ of the $3 \mathrm{D}$-amplified pulse at different radii $\rho$ and for $z=5 \mathrm{~mm}$. As expected, the soft $\mathrm{x}$-ray field is more intense for some trajectories, for which the intensity can reach saturation. For these trajectories the temporal modulations (e.g. the trajectory corresponding to a radius of $15 \mu \mathrm{m}$ ) begins to be observable but they remain weak due to the limited amplification length $(<4 \mathrm{~mm})$. On most part of the other trajectories, the integrated gain is much smaller, so that, when summing all the trajectories over the radius, the Rabi oscillations have a negligible contribution, as observed on Fig. 2-(b). In addition, these trajectory modulations are not in phase with each other since $\Omega_{R}$ depends on the resonant field intensity, and will be furthered dampened when integrated.

Reaching the oscillatory regime would allow measurements of intimate plasma parameters, and could be a very promising approach to produce high flux sub-ps pulses by favoring the first oscillation peak. Our calculations show that this regime is reached when the amplified field intensity is higher than the saturation intensity of the amplifier. In order to get an observable effect, this condition has to be satisfied by the majority of the trajectories followed by the injected $\mathrm{HOH}$ signal. The amplifier is also needed to be as homogeneous as possible. The standard OFI setup will not allow the generation of a homogeneous, long enough amplifier. Assuming the use of a reasonable (multi-terawatt) laser installation, that can be achieved in an OFI plasma by guiding the pump laser over a long distance. Plasma waveguiding $^{202122}$ is considered a serious method towards this goal.

Using a 3D modeling of an optical-field-ionized harmonic-seeded soft x-ray laser coupled 
with a 3D Maxwell-Bloch amplification treatment, we have shown that the temporal structure of the output pulse does not exhibit any features characteristic of Rabi oscillations of the population inversion. This is mainly due to the amplifier inhomogeneity and short effective length induced by plasma dispersion. Strong Rabi oscillations that can be efficiently used to create a sub-ps pulse of very high intensity could nonetheless be generated by longer and more uniform plasma targets. A promising approach to achieve such kind of amplifiers would be to guide the pump beam at relatively moderate intensity over $1 \mathrm{~cm}$ or more inside a high-density krypton plasma. 


\section{REFERENCES}

${ }^{1}$ T. Ditmire, M. H. R. Hutchinson, M. H. Key, C. L. S. Lewis, A. MacPhee, I. Mercer, D. Neely, M. D. Perry, R. A. Smith, J. S. Wark, and M. Zepf, "Amplification of xuv harmonic radiation in a gallium amplifier," Phys. Rev. A 51, R4337-R4340 (1995).

${ }^{2}$ P. Zeitoun, G. Faivre, S. Sebban, T. Mocek, A. Hallou, M. Fajardo, D. Aubert, P. Balcou, F. Burgy, D. Douillet, S. Kazamias, G. de Lacheze-Murel, T. Lefrou, S. le Pape, P. Mercere, H. Merdji, A. S. Morlens, J. P. Rousseau, and C. Valentin, "A high-intensity highly coherent soft x-ray femtosecond laser seeded by a high harmonic beam," Nature 431, 426-429 (2004).

${ }^{3}$ Y. Wang, E. Granados, M. A. Larotonda, M. Berrill, B. M. Luther, D. Patel, C. S. Menoni, and J. J. Rocca, "High-brightness injection-seeded soft-x-ray-laser amplifier using a solid target," Phys. Rev. Lett. 97, 123901 (2006).

${ }^{4}$ Y. Wang, M. Berrill, F. Pedaci, M. M. Shakya, S. Gilbertson, Z. Chang, E. Granados, B. M. Luther, M. A. Larotonda, and J. J. Rocca, "Measurement of 1-ps soft-x-ray laser pulses from an injection-seeded plasma amplifier," Phys. Rev. A 79, 023810 (2009).

${ }^{5}$ O. Guilbaud, F. Tissandier, J.-P. Goddet, M. Ribière, S. Sebban, J. Gautier, D. Joyeux, D. Ros, K. Cassou, S. Kazamias, A. Klisnick, J. Habib, P. Zeitoun, D. Benredjem, T. Mocek, J. Nedjl, S. de Rossi, G. Maynard, B. Cros, A. Boudaa, and A. Calisti, "Fourierlimited seeded soft x-ray laser pulse," Opt. Lett. 35, 1326-1328 (2010).

${ }^{6}$ F. Pedaci, Y. Wang, M. Berrill, B. Luther, E. Granados, and J. J. Rocca, "Highly coherent injection-seeded 13.2 nm tabletop soft x-ray laser," Opt. Lett. 33, 491-493 (2008).

${ }^{7}$ Y. Wang, E. Granados, F. Pedaci, D. Alessi, B. Luther, M. Berrill, and J. J. Rocca, "Phase-coherent, injection-seeded, table-top soft-x-ray lasers at $18.9 \mathrm{~nm}$ and $13.9 \mathrm{~nm}, "$ Nat. Photon. 2, 94-98 (2008).

${ }^{8}$ J. A. Koch, B. J. MacGowan, L. B. Da Silva, D. L. Matthews, J. H. Underwood, P. J. Batson, R. W. Lee, R. A. London, and S. Mrowka, "Experimental and theoretical investigation of neonlike selenium x-ray laser spectral linewidths and their variation with amplification," Phys. Rev. A 50, 1877-1898 (1994).

${ }^{9}$ I. R. Al'miev, O. Larroche, D. Benredjem, J. Dubau, S. Kazamias, C. Möller, and A. Klisnick, "Dynamical description of transient x-ray lasers seeded with high-order harmonic radiation through maxwell-bloch numerical simulations," Phys. Rev. Lett. 99, 123902 (2007). 
${ }^{10}$ O. Larroche, D. Ros, A. Klisnick, A. Sureau, C. Möller, and H. Guennou, "Maxwell-bloch modeling of x-ray-laser-signal buildup in single- and double-pass configurations," Phys. Rev. A 62, 043815 (2000).

${ }^{11}$ C. M. Kim, J. Lee, and K. A. Janulewicz, "Coherent amplification of an ultrashort pulse in a high- and swept-gain medium with level degeneracy," Phys. Rev. Lett. 104, 053901 (2010).

${ }^{12}$ A. E. Siegman, Lasers, ISBN 0-935702-11-3 (University Science Books, 1986).

${ }^{13}$ B. Cros, T. Mocek, I. Bettaibi, G. Vieux, M. Farinet, J. Dubau, S. Sebban, and G. Maynard, "Characterization of the collisionally pumped optical-field-ionized soft-x-ray laser at 41.8nm driven in capillary tubes," Phys. Rev. A 73, 033801 (2006).

${ }^{14}$ B. Robillart, G. Maynard, B. Cros, A. Boudaa, J. Dubau, S. Sebban, and J.-P. Goddet, "Modeling of an ultra-short x-ray laser pulse amplification through an optical-field-ionized gas using a maxwell-bloch treatment," in X-Ray Lasers 2008, Springer Proceedings in Physics, Vol. 130, edited by C. L. S. Lewis and D. Riley (Springer, 2009) pp. 255-262.

${ }^{15}$ F. Kärtner, "Ultrafast optics," MIT Open Courseware (2005).

${ }^{16}$ F. Tissandier, S. Sebban, M. Ribière, J. Gautier, P. Zeitoun, G. Lambert, A. Barszczak Sardinha, J.-P. Goddet, F. Burgy, T. Lefrou, C. Valentin, A. Rousse, O. Guilbaud, A. Klisnick, J. Nejdl, T. Mocek, and G. Maynard, "Observation of spectral gain narrowing in a high-order harmonic seeded soft-x-ray amplifier," Phys. Rev. A 81, 063833 (2010).

${ }^{17}$ F. Tissandier, S. Sebban, M. Ribière, J. Gautier, P. Zeitoun, G. Lambert, J.-P. Goddet, F. Burgy, C. Valentin, A. Rousse, J. Nejdl, T. Mocek, and G. Maynard, "Bessel spatial profile of a soft x-ray laser beam," Appl. Phys. Lett. 97, 231106 (2010).

${ }^{18}$ C. M. Kim, K. A. Janulewicz, H. T. Kim, and J. Lee, "Amplification of a high-order harmonic pulse in an active medium of a plasma-based x-ray laser," Phys. Rev. A 80, 053811 (2009).

${ }^{19}$ L. M. Meng, D. Alessi, O. Guilbaud, Y. Wang, M. Berrill, B. Luther, S. R. Domingue, D. H. Martz, D. Joyeux, S. D. Rossi, J. J. Rocca, and A. Klisnick, "Temporal coherence and spectral linewidth of an injection-seeded transient collisional soft x-ray laser," Opt. Express 19, 12087-12092 (2011).

${ }^{20}$ A. Butler, A. J. Gonsalves, C. M. McKenna, D. J. Spence, S. M. Hooker, S. Sebban, T. Mocek, I. Bettaibi, and B. Cros, "Demonstration of a collisionally excited optical-field- 
ionization xuv laser driven in a plasma waveguide," Phys. Rev. Lett. 91, 205001 (2003). ${ }^{21}$ A. Butler, A. J. Gonsalves, C. M. McKenna, D. J. Spence, S. M. Hooker, S. Sebban, T. Mocek, I. Betttaibi, and B. Cros, "41.8-nm xe ${ }^{8+}$ laser driven in a plasma waveguide," Phys. Rev. A 70, 023821 (2004).

${ }^{22}$ M.-C. Chou, P.-H. Lin, C.-A. Lin, J.-Y. Lin, J. Wang, and S.-Y. Chen, "Dramatic enhancement of optical-field-ionization collisional-excitation x-ray lasing by an optically preformed plasma waveguide," Phys. Rev. Lett. 99, 063904 (2007). 

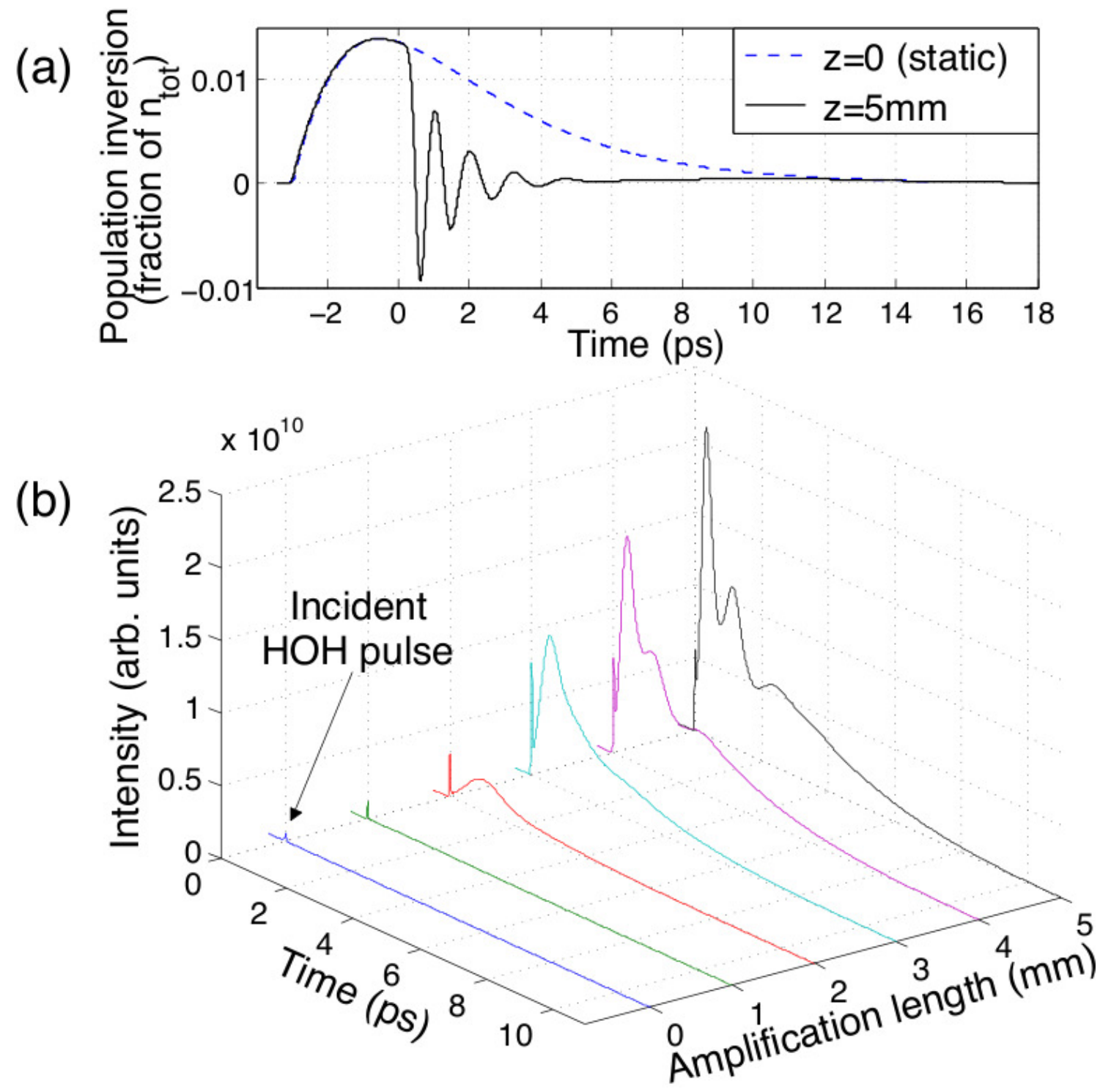

FIG. 1. (Color online) (a) Temporal evolution of the population inversion in the plasma without applied field (dashed line) and after an amplification length of 5mm (solid line). (b) Temporal profile of the resonant XUV field along its amplification. The $\mathrm{HOH}$ pulse is injected at $t=0,3 \mathrm{ps}$ after OFI. 
(a)

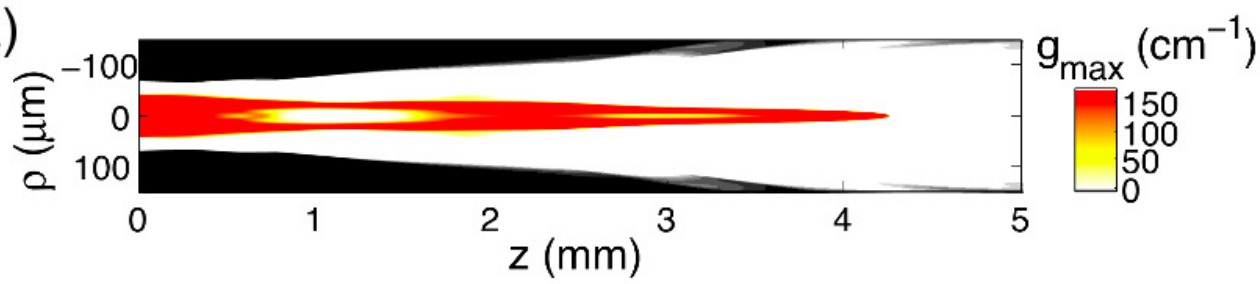

(b)

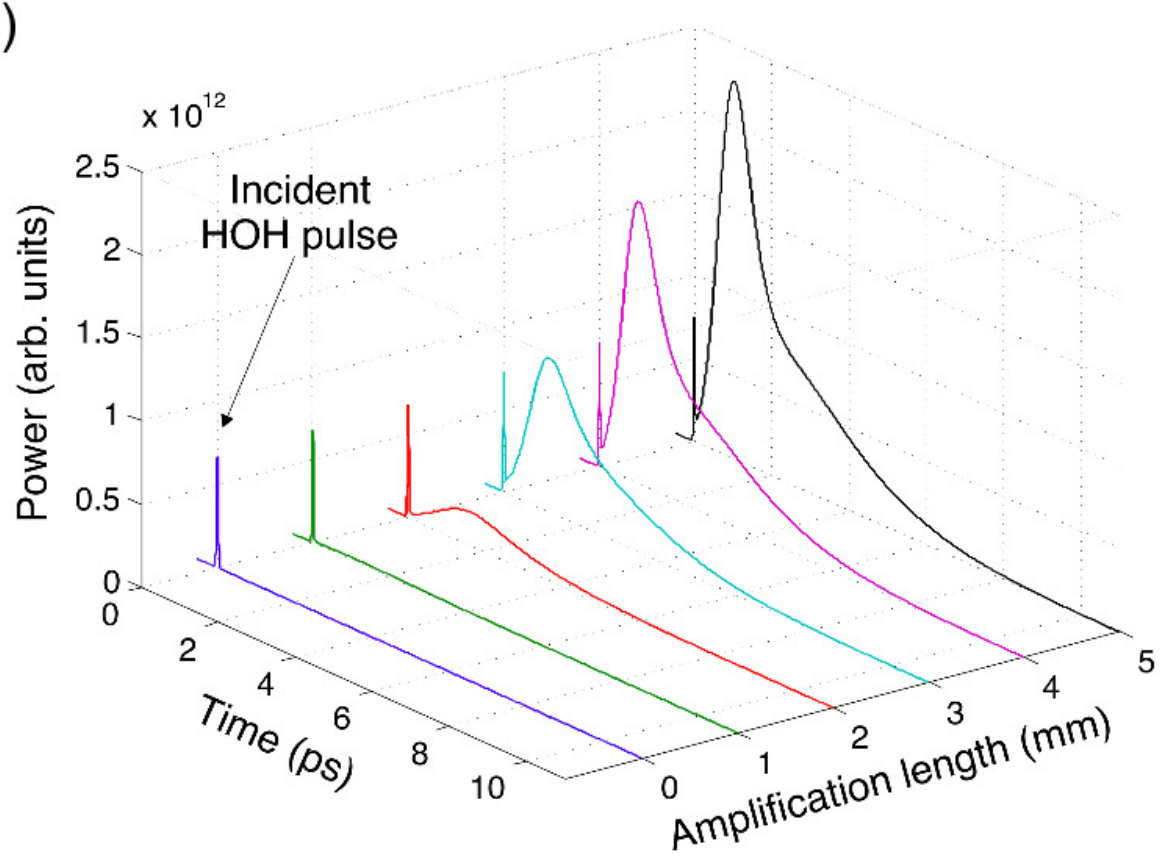

FIG. 2. (Color online) (a) Calculated 2D gain map of the amplifier. The infrared pump laser propagates from left to right. The white-colored region represents transparent medium at the resonance wavelength while the black-colored one is absorbing. (b) 3D calculation of the temporal profile of the resonant XUV field along its amplification in the plasma. 


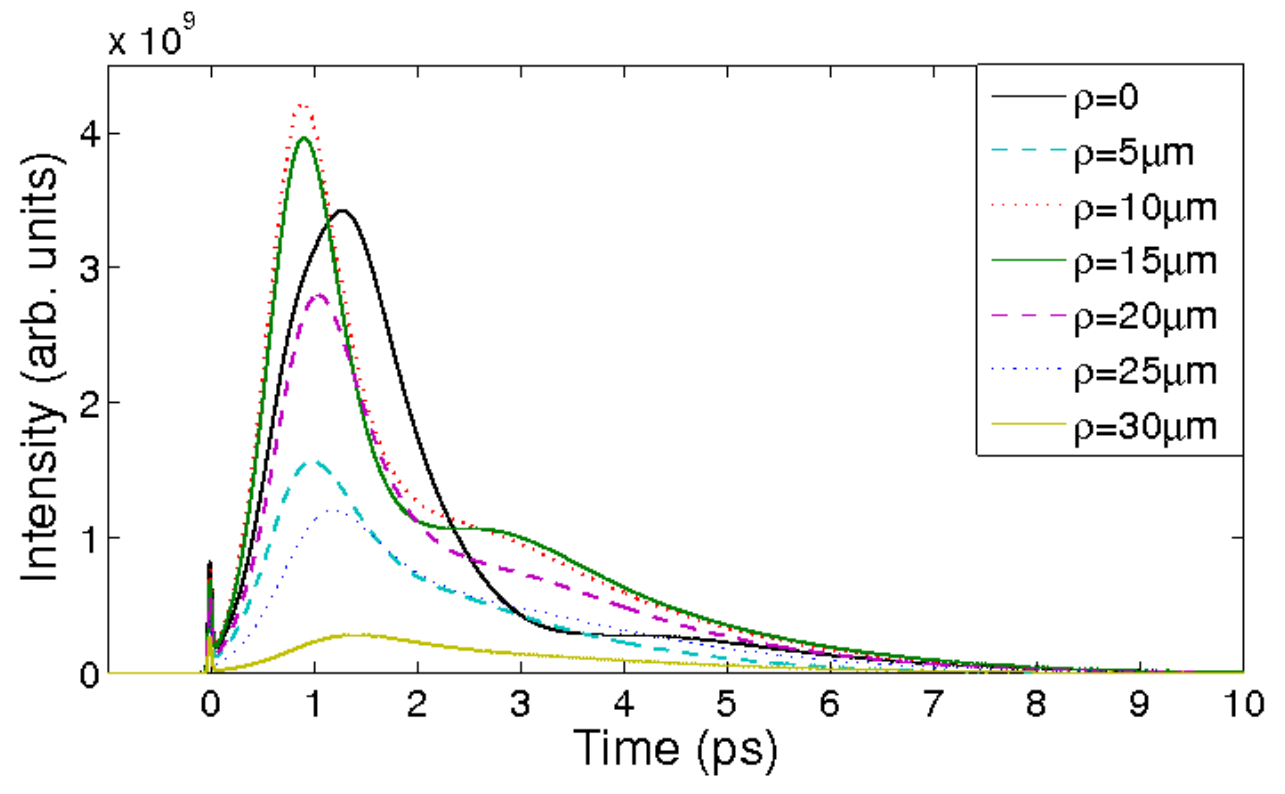

FIG. 3. (Color online) Temporal profile of the output XUV pulse for different linear trajectories at different radii, calculated using the whole plasma length. 